\section{Atividade física e alimentação saudável em escolares brasileiros: revisão de programas de intervenção}

\author{
Physical activity and healthy eating in Brazilian \\ students: a review of intervention programs
}

\author{
1 Faculdade de Educação \\ Física, Universidade de \\ Brasília, Brasília, Brasil. \\ 2 Programa de Pós-graduação \\ em Educação Física \\ Universidade Federal do \\ Paraná, Curitiba, Brasil. \\ 3 Escola Superior \\ de Educação Física, \\ Universidade Federal de \\ Pelotas, Pelotas, Brasil. \\ Correspondência \\ E. A. Souza \\ Faculdade de Educação Física, \\ Universidade de Brasília. \\ Campus Universitário Darcy \\ Ribeiro, Asa Norte, Brasília, \\ DF 70000-000, Brasil. \\ profeas@gmail.com
}

\section{Abstract}

This article provides a systematic literature review on physical activity and/or healthy eating interventions among Brazilian students. Complete articles published from 2004 to 2009 were searched in the SciELO, MEDLINE, and CAPES electronic databases, in the articles' references, and through contacts with authors. Six studies covered nutritional interventions, another six analyzed nutrition and physical activity, and one discussed changes in body composition. Interventions produced different results according to their objectives: increase in weekly physical activity; improvement in eating habits and knowledge on nutrition; and decrease in overweight and obesity. School health promotion programs are essential for raising awareness on the relevance of health promotion and the adoption of healthy habits. However, further longitudinal studies are needed to produce evidence on sustainability of programs and healthy habits.

Motor Activity; Public Health Nutrition; Students
Evanice Avelino de Souza 1 Valter Cordeiro Barbosa Filho 2 Júlia Aparecida Devidé Nogueira 1 Mario Renato de Azevedo Júnior 3

\section{Introdução}

A infância e a adolescência são períodos extremamente importantes para o desenvolvimento de um estilo de vida saudável, uma vez que os comportamentos adquiridos nesta fase tendem a ser perpetuados por toda a vida ${ }^{1}$. Durante a adolescência também ocorrem o aumento da independência e ganho de autonomia na tomada de decisões sobre práticas e comportamentos de vida ${ }^{2}$. Essa situação pode ser preocupante pelo fato de que os adolescentes passam a ficar mais expostos a comportamentos de risco como etilismo, tabagismo, sedentarismo e alimentação inadequada.

Estudos nacionais e internacionais sobre fatores de risco e de proteção comportamentais relacionados à saúde em adolescentes, como o Global School-based Student Health Survey, o Youth Health Risk Beahaviour Surveillance System, o Health Behaviour in School-aged Children Study 2 e a Pesquisa Nacional de Saúde do Escolar ${ }^{3}$, mostram que o estilo de vida adotado por crianças e adolescentes não é saudável, incluindo baixo consumo de frutas, inatividade física, inabilidade de manter um peso corporal saudável e consumo de bebidas alcoólicas e tabaco. Esses comportamentos de risco à saúde estão cada vez mais presentes na sociedade contemporânea e estão associados ao desenvolvimento das doenças crônicas não transmissíveis (DCNT). As DCNT estão aumentando no público jovem, lideram as cau- 
sas de morbi-mortalidade no Brasil e no mundo, e acarretam graves impactos pessoais, sociais e financeiros 4,5 .

A atividade física e a alimentação são dois comportamentos considerados prioritários para a promoção da saúde e prevenção de DCNT em populações contemporâneas 4 . A promoção de hábitos saudáveis em crianças e adolescentes possui relevância estratégica e deve ser encarada como prioridade por todos os setores sociais. Por congregar a maioria das crianças e adolescentes de um país, a escola representa um espaço privilegiado para o desenvolvimento dessas ações 6,7,8,9.

No Brasil, existem algumas intervenções destinadas à promoção de atividade física e alimentação saudável em escolares 4,10. Entretanto, pouco se conhece sobre a metodologia dos estudos, os tipos de intervenção, as evidências dos efeitos e os resultados na saúde dos alunos. Nesse contexto, o objetivo do presente estudo foi revisar sistematicamente a literatura sobre intervenções de promoção da prática de atividade física e/ou alimentação saudável, realizadas entre escolares brasileiros, e sumarizar seus principais resultados.

\section{Métodos}

Esta revisão sistemática buscou identificar artigos publicados entre janeiro de 2004 e dezembro de 2009, considerando as bases de dados SciELO (http://www.scielo.org) eMEDLINE (http://www. ncbi.nlm.nih.gov/pubmed). Além dessas bases de dados foi realizada busca de dissertações e teses no portal da Coordenação de Aperfeiçoamento de Pessoal de Nível Superior - CAPES (http:// capesdw.capes.gov.br/capesdw/). Os seguintes descritores e suas combinações em português e inglês foram utilizados para a busca: programas de saúde, promoção de saúde, escolares, crianças, adolescentes, intervenção, atividade física, nutrição, hábitos alimentares e obesidade. Além disso, as referências bibliográficas dos estudos encontrados também foram pesquisadas a fim de localizar mais estudos sobre o tema.

Foram selecionados os estudos que atenderam aos seguintes critérios de inclusão: (i) os publicados em periódicos, dissertações e teses disponíveis nas bases de dados pesquisadas; (ii) amostra que incluíssem crianças e adolescentes; e (iii) intervenções realizadas em escolas públicas e/ou privadas. Não foram incluídos estudos com: (i) amostra exclusiva de crianças e/ou adolescentes em condições específicas de saúde (obesos, hipertensos, diabéticos etc.); (ii) faixa etária que incluísse apenas crianças pré- escolares ( $<6$ anos) ou adultos ( $>18$ anos); (iii) intervenção realizada totalmente fora do ambiente escolar (clubes, comunidades, clínicas); (iv) intervenção realizada unicamente com professores; e (v) artigos com descrição exclusiva do delineamento metodológico de programas de intervenção. As características da amostra e das intervenções realizadas pelos estudos selecionados, bem como seus principais resultados, foram tabulados e analisados criticamente. Os estudos foram apresentados nas tabelas em ordem crescente do ano de publicação e, quando do mesmo ano, em ordem alfabética considerando o primeiro autor.

\section{Resultados}

Dezessete estudos foram inicialmente encontrados e, destes, 13 atenderam aos critérios de inclusão desta revisão. Três estudos foram excluídos por terem sido realizados com crianças e adolescentes obesos e um foi excluído por não estar disponível na íntegra no banco de dados pesquisado e, mesmo buscando contato com o autor, não obtivemos resposta.

Dos 13 estudos revisados, foram encontrados sete artigos 11,12,13,14,15,16,17, três dissertações $18,19,20$, duas teses 21,22 e um relatório 23 . As características gerais dos trabalhos como autoria, local e ano de execução, faixa etária da população, número amostral e tipo da escola estudada estão descritas na Tabela 1.

Seis estudos foram realizados na Região Sudeste 11,12,14,18,20,22 (dois em Niterói, Rio de Janeiro; dois em Ribeirão Preto e dois em São Paulo, São Paulo), quatro na Região Sul 13,17,21,23 (três em Florianópolis, Santa Catarina; e um em Pelotas, Rio Grande do Sul), um na Região Norte 16 (Porto Velho, Rondônia), um na Região Centro-oeste 19 (Distrito Federal) e um estudo realizado simultaneamente nas regiões Sul e Nordeste 15 (Florianópolis e Recife, respectivamente).

Todos os estudos trabalharam com meninos e meninas simultaneamente; a faixa etária pesquisada esteve entre 6 e 24 anos de idade; o número amostral dos estudos variou entre $34 \mathrm{e}$ 2.209 escolares, com a maioria 11,12,15,19,20,21,22,23 das intervenções realizada em escolas públicas. Apenas três 14,16,23 intervenções reportaram o nível socioeconômico dos participantes, onde um estudo 14 foi realizado com escolares de baixo nível socioeconômico e os demais tiveram a participação de estudantes de diversos níveis socioeconômicos, de A-C 16 e de A-E 23. Quanto ao tipo da amostra, cinco (30,8\%) estudos tiveram seleção aleatória ou das escolas 11,15,23 ou das turmas de ensino 14,21 que realizaram intervenção. 
Características dos estudos de intervenção realizados em escolas brasileiras (2004-2009).

\begin{tabular}{|c|c|c|c|c|c|c|}
\hline Referência & Cidade (Estado) & Ano da intervenção & Escolas & Ensino & Faixa etária (anos) & Amostra \\
\hline Barros 21 & $\begin{array}{l}\text { Florianópolis } \\
\text { (Santa Catarina) }\end{array}$ & 2003 & $\begin{array}{l}3 \text { públicas }(\mathrm{Gl}) ; \\
3 \text { públicas }(\mathrm{GC})\end{array}$ & Ensino Médio & $15-19$ & $\begin{array}{c}\mathrm{GI}=452 \\
(53,1 \% \text { meninas }) \\
\mathrm{GC}=300 \\
(53,7 \% \text { meninas })\end{array}$ \\
\hline Gaglianone et al. 11 & $\begin{array}{l}\text { São Paulo } \\
\text { (São Paulo) }\end{array}$ & 2000 & $\begin{array}{l}3 \text { públicas }(\mathrm{Gl}) ; \\
5 \text { públicas }(\mathrm{GC})\end{array}$ & $\begin{array}{l}\text { 1ạ e 2a séries do } \\
\text { Ensino Fundamental }\end{array}$ & $7-10$ & $\begin{array}{l}\mathrm{GI}=294(\mathrm{NI}) \\
\mathrm{GC}=343(\mathrm{NI})\end{array}$ \\
\hline Menezes et al. 23 & $\begin{array}{c}\text { Pelotas } \\
\text { (Rio Grande do Sul) }\end{array}$ & 2004 & $\begin{array}{l}16 \text { públicas }(\mathrm{Gl}) ; \\
16 \text { públicas }(\mathrm{GC})\end{array}$ & $\begin{array}{l}\text { 7ạ e 8a séries do } \\
\text { Ensino Fundamental }\end{array}$ & $13-15$ & $\begin{array}{c}\mathrm{Gl}=955 \\
(55,6 \% \text { meninas }) ; \\
\mathrm{GC}=1.146 \\
(55,7 \% \text { meninas })\end{array}$ \\
\hline Deminice et al. 12 & $\begin{array}{l}\text { Ribeirão Preto } \\
\text { (São Paulo) }\end{array}$ & 2005 & 1 pública & $\begin{array}{c}\text { Pré-escola a } \\
\text { 8a série do Ensino } \\
\text { Fundamental }\end{array}$ & $6-16$ & $\mathrm{GI}=951$ \\
\hline Gabriel et al. 13 & $\begin{array}{l}\text { Florianópolis } \\
\text { (Santa Catarina) }\end{array}$ & 2004 & $\begin{array}{l}1 \text { pública e } \\
1 \text { privada }\end{array}$ & $\begin{array}{c}\text { 3a e 4a séries do } \\
\text { Ensino Fundamental }\end{array}$ & $9-11$ & $\begin{array}{c}\mathrm{GI}=162 \\
(54,9 \% \text { meninas })\end{array}$ \\
\hline Sichieri et al. 14 & $\begin{array}{c}\text { Niterói } \\
\text { (Rio de Janeiro) }\end{array}$ & 2005 & $\begin{array}{c}22 \text { turmas (GI); } \\
24 \text { turmas (GC), de } \\
22 \text { escolas públicas }\end{array}$ & $\begin{array}{l}\text { 4a série do Ensino } \\
\text { Fundamental }\end{array}$ & $9-12$ & $\begin{array}{c}\mathrm{GI}=434 \\
(46,9 \% \text { meninas }) \\
\mathrm{GC}=608 \\
(47,4 \% \text { meninas })\end{array}$ \\
\hline Vargas 18 & $\begin{array}{c}\text { Niterói } \\
\text { (Rio de Janeiro) }\end{array}$ & 2005 & $\begin{array}{l}1 \text { pública }(G I) ; \\
1 \text { pública }(G C)\end{array}$ & $\begin{array}{l}\text { 5a e 6a séries do } \\
\text { Ensino Fundamental }\end{array}$ & $11-17$ & $\begin{array}{c}\mathrm{GC}=99 \\
(52,1 \% \text { meninas }) ; \\
\mathrm{GI}=162 \\
(53,5 \% \text { meninas })\end{array}$ \\
\hline Zancul 22 & $\begin{array}{l}\text { Ribeirão Preto } \\
\text { (São Paulo) }\end{array}$ & $2006 / 2007$ & 1 pública & $\begin{array}{l}\text { 6a série do Ensino } \\
\text { Fundamental }\end{array}$ & $11-14$ & $\begin{array}{c}\mathrm{Gl}=36 \\
(47,2 \% \text { meninas })\end{array}$ \\
\hline Barros et al. 15 & $\begin{array}{c}\text { Florianópolis } \\
\text { (Santa Catarina); } \\
\text { Recife (Pernambuco) }\end{array}$ & 2006 & $\begin{array}{l}10 \text { públicas (GI); } \\
10 \text { públicas (GC) }\end{array}$ & $\begin{array}{l}\text { Ensino Médio } \\
\text { noturno }\end{array}$ & $15-24$ & $\begin{array}{l}\mathrm{GI}=474(\mathrm{NI}) \\
\mathrm{GC}=515(\mathrm{NI})\end{array}$ \\
\hline Cavalcanti 19 & Distrito Federal & 2008 & $\begin{array}{l}1 \text { pública (GI); } \\
1 \text { pública (GC) }\end{array}$ & Ensino Fundamental & $7-11$ & $\begin{array}{l}\mathrm{GI}=229(\mathrm{NI}) \\
\mathrm{GC}=232(\mathrm{NI})\end{array}$ \\
\hline Farias et al. 16 & $\begin{array}{l}\text { Porto Velho } \\
\text { (Rondônia) }\end{array}$ & 2006 & $\begin{array}{l}1 \text { privada }(\mathrm{Gl}) ; \\
1 \text { privada }(\mathrm{GC})\end{array}$ & Ensino Fundamental & $10-15$ & $\begin{array}{c}\mathrm{Gl}=186 \\
(48,4 \% \text { meninas }) \\
\mathrm{GC}=197 \\
(45,2 \% \text { meninas })\end{array}$ \\
\hline Fernandes et al. 17 & $\begin{array}{l}\text { Florianópolis } \\
\text { (Santa Catarina) }\end{array}$ & 2009 & $\begin{array}{l}1 \text { pública e } \\
1 \text { privada }\end{array}$ & $\begin{array}{l}\text { 2a série do Ensino } \\
\text { Fundamental }\end{array}$ & 8,2 (média) & $\begin{array}{c}\mathrm{Gl}=55 \\
(61,8 \% \text { meninas }) \\
\mathrm{GC}=80 \\
(46,2 \% \text { meninas })\end{array}$ \\
\hline Ribeiro 20 & $\begin{array}{l}\text { São Paulo } \\
\text { (São Paulo) }\end{array}$ & 2008 & $\begin{array}{l}1 \text { pública }\left(G I_{1}\right) ; \\
1 \text { pública }\left(G I_{2}\right) ; \\
1 \text { pública }(G C)\end{array}$ & $\begin{array}{l}\text { 7ạ série do Ensino } \\
\text { Fundamental. }\end{array}$ & $12-14$ & $\begin{array}{c}\mathrm{Gl}_{1}=25 \\
(48,0 \% \text { meninas }) ; \\
\mathrm{Gl}_{2}=23 \\
(52,2 \% \text { meninas }) ; \\
\mathrm{GC}=21 \\
(42,9 \% \text { meninas })\end{array}$ \\
\hline
\end{tabular}

GC: grupo controle; $\mathrm{Gl}$ : grupo intervenção. $\mathrm{Gl}_{1}$ : intervenção sem prática de atividade física; $\mathrm{Gl}_{2}$ : intervenção com atividade física; $\mathrm{Nl}$ : não indicado. 
Entretanto, os demais apresentaram seleção dos participantes por conveniência.

Na Tabela 2 foram descritos os objetivos gerais, os principais resultados e características dos estudos inseridos nesta revisão. Verificouse que a duração das intervenções nos estudos variou entre dois 13 a 1116 meses. Quanto aos objetivos das intervenções, seis trabalhos 11,12,13,14,17,22 intervieram focando a mudança no conhecimento nutricional, práticas alimentares e/ou classificação do estado nutricional; outros seis 15,18,19,20,21,23 focaram tanto as mudanças na alimentação quanto na prática de atividade física, enquanto um estudo 16 realizou intervenção visando a modificações no estado nutricional (sobrepeso/obesidade) e na composição corporal. Quanto às variáveis analisadas, a maioria dos estudos 13,14,17,18,19,20,22,23 utilizou o índice de massa corporal (IMC) como indicador de sobrepeso e/ou obesidade, enquanto apenas um 21 avaliou o impacto e as ações desenvolvidas na intervenção.

Alguns resultados devem ser destacados nos trabalhos que focaram mudanças no conhecimento/prática de atividade física e nutrição, a saber: aumento na quantidade (minutos/dia) de prática semanal de atividade física 20; maior animação para a prática de atividade física 18; e redução do consumo de alimentos com alto valor calórico (doces, biscoitos, salgados) 18,19. Nos estudos que tiveram somente os componentes alimentares e/ou classificação do estado nutricional como foco da intervenção, os principais resultados foram a redução do consumo e da preferência por alimentos de alto valor calórico $11,13,14,17$, melhoria no conhecimento sobre alimentação 12 e nas práticas alimentares saudáveis (consumo diário de desjejum e a ingestão de verduras no almoço e jantar) 22 . O programa de intervenção 16 que focou nos indicadores antropométricos e na composição corporal verificou a redução da prevalência de sobrepeso e obesidade, como seu principal resultado.

\section{Programas de intervenção focados na} alimentação saudável e no estado nutricional

Dentre os seis programas de intervenção 11,12, 13,14,17,22 realizados com enfoque na promoção da alimentação saudável e do estado nutricional dos escolares brasileiros, podem ser destacadas três ações de intervenção como as mais utilizadas na discussão da importância da alimentação para a saúde na infância e adolescência: jogos e histórias sobre nutrição, apresentação de vídeos e palestras com os escolares, e a distribuição e/ou confecção de material educativo acerca da alimentação saudável. Essas atividades foram desenvolvidas nos seis programas de intervenção 11,12,13,14,17,22 e, juntamente com outras ações de intervenção, buscaram promover o conhecimento e/ou atitudes positivas relacionadas à alimentação, abordando temas como a importância dos alimentos em diversas funções do organismo 11,12,13,14,17, valor nutricional dos alimentos 12,13,14,17,22 e a montagem de refeições e lanches conforme recomendações alimentares para crianças e adolescentes, como as propostas pelo Guia da Pirâmide Alimentar 13,17,22.

Um importante programa de intervenção que desenvolveu essas ações foi realizado com 434 escolares (608 no grupo controle - GC) da rede pública de ensino de Niterói 14. Esse programa de intervenção teve a duração de sete meses e focou, com base na realização de jogos, quizzes, competições com músicas e desenhos, $\mathrm{e}$ distribuição de material personalizado, mensagens relacionadas sobre a importância da água para a saúde e a substituição de bebidas gaseificadas pelo consumo de água. A partir dessas ações de intervenção, foi verificada redução significativa do consumo de bebidas gaseificadas entre os escolares do grupo intervenção (GI) em comparação ao controle (média da diferença = -56mL; IC95\%: -119; -7mL). Entretanto, o IMC e os demais componentes da alimentação analisados (consumo de frutas, adição de açúcar em bebidas, entre outros) não tiveram alterações significativas 14 .

A mudança nos hábitos alimentares dos escolares foi um dos resultados mais reportados pelos programas de intervenção, principalmente com a redução do consumo de alimentos de alto valor calórico (refrigerantes, bolachas recheadas e suco artificial) 11,13,14,17 e o aumento do consumo de alimentos saudáveis (por exemplo, frutas e verduras) $11,13,22$. Um programa de intervenção que caracteriza essas evidências foi realizado por Fernandes et al. 17, com 55 escolares (80 no GC) da cidade de Florianópolis. Após os quatro meses de intervenção, foi observada a redução de 4,81\% ( $\mathrm{p}=0,01)$ da proporção de crianças que consomem frequentemente ( 2 ou 3 dias na semana) suco artificial na escola, produto com venda proibida por lei nos colégios do Estado de Santa Catarina. Outros alimentos proibidos, como salgadinho industrializado e refrigerante, tiveram aumento no consumo semanal entre os escolares do GC, o que não ocorreu no GI 17.

A continuidade dos hábitos alimentares saudáveis após o programa de intervenção foi avaliada somente em um estudo 22 . Nesse programa, foi verificado o aumento de 30,9\% (p=0,02) e $21,5 \%(p=0,02)$ na proporção de escolares que referiram consumir verduras cruas no almoço e no jantar, respectivamente, após seis meses de 
Tabela 2

Descrição dos estudos de intervenção em escolas brasileiras (2004-2009) de acordo com o objetivo, características da intervenção, variáveis e principais resultados.

\begin{tabular}{|c|c|c|c|c|}
\hline Referência & Objetivo & Características da intervenção & Variáveis & Principais resultados \\
\hline Barros 21 & $\begin{array}{l}\text { Avaliar a implementação de } \\
\text { uma intervenção que visa a } \\
\text { promover atividade física e } \\
\text { padrões saudáveis entre os } \\
\text { estudantes }\end{array}$ & $\begin{array}{c}\text { Duração: } \pm 3 \text { meses. } \\
\text { Descrição: atividades } \\
\text { educacionais, organizacionais } \\
\text { e ambientais baseadas no } \\
\text { modelo lógico das Escolas } \\
\text { Promotoras de Saúde. Foco } \\
\text { na modificação ambiental } \\
\text { e normativa, educação e } \\
\text { treinamento e engajamento de } \\
\text { pessoal }\end{array}$ & $\begin{array}{c}\text { Instrumento: questionário } \\
\text { desenvolvido para o estudo. } \\
\text { Variáveis analisadas: impacto e } \\
\text { ações da intervenção, prática } \\
\text { de atividade física e hábitos } \\
\text { alimentares }\end{array}$ & $\begin{array}{c}\text { Aumento: } 33,1 \% \text { das meninas } \\
\text { estavam mais informadas } \\
\text { sobre hábitos alimentares após } \\
\text { a intervenção. Redução: 11,8\% } \\
\text { na proporção de meninos que } \\
\text { consideravam a textura/sabor } \\
\text { como barreira para consumo } \\
\text { de alimentos }\end{array}$ \\
\hline Gaglianone et al. 11 & $\begin{array}{c}\text { Analisar o impacto do } \\
\text { programa de educação } \\
\text { nutricional nos conhecimentos } \\
\text { e atitudes sobre hábitos } \\
\text { alimentares saudáveis }\end{array}$ & $\begin{array}{c}\text { Duração: } 14 \text { semanas. } \\
\text { Descrição: treinamento de } \\
\text { professores para orientar os } \\
\text { escolares sobre alimentação } \\
\text { saudável. Dinâmicas } \\
\text { psicossociais com os escolares } \\
\text { com base na utilização de } \\
\text { materiais e jogos educacionais }\end{array}$ & $\begin{array}{l}\text { Instrumento: questionário } \\
\text { desenvolvido para o estudo. } \\
\text { Variáveis analisadas: nível de } \\
\text { conhecimento em alimentação } \\
\text { e nutrição e hábitos } \\
\text { alimentares }\end{array}$ & $\begin{array}{c}\text { Aumento: } 13,8 \% \text { na proporção } \\
\text { de escolares do Gl que } \\
\text { consumiam frequentemente } \\
\text { todos os grupos alimentares } \\
\text { recomendados na Pirâmide } \\
\text { Alimentar. Redução: } 24,3 \% \\
\text { na preferência dos escolares } \\
\text { por alimentos com alto valor } \\
\text { calórico (alimentos ricos em } \\
\text { gordura e açucares simples) }\end{array}$ \\
\hline Menezes et al. 23 & $\begin{array}{l}\text { Avaliar o efeito do programa } \\
\text { de intervenção educacional } \\
\text { sobre o tabagismo, hábitos } \\
\text { alimentares, atividade física e } \\
\text { IMC dos escolares }\end{array}$ & $\begin{array}{l}\text { Duração: } 6 \text { meses. Descrição: } \\
\text { treinamento de professores } \\
\text { com distribuição de fôlderes, } \\
\text { cartazes e vídeos que } \\
\text { deveriam ser repassados para } \\
\text { os escolares }\end{array}$ & $\begin{array}{c}\text { Instrumentos: questionário } \\
\text { desenvolvido para o estudo } \\
\text { e avaliação antropométrica. } \\
\text { Variáveis analisadas: } \\
\text { IMC, conhecimento e } \\
\text { comportamento sobre } \\
\text { tabagismo, NAF e hábitos } \\
\text { alimentares }\end{array}$ & $\begin{array}{c}\text { O NAF, IMC e hábitos } \\
\text { alimentares não apresentaram } \\
\text { mudança significativa após } \\
\text { intervenção. Aumento: } 12,6 \% \\
\text { no percentual de adolescentes } \\
\text { que consideram perigoso o } \\
\text { fumo passivo }\end{array}$ \\
\hline Deminice et al. 12 & $\begin{array}{c}\text { Avaliar os efeitos de um } \\
\text { programa de educação } \\
\text { alimentar sobre alimentação, } \\
\text { práticas alimentares e o estado } \\
\text { nutricional dos escolares }\end{array}$ & $\begin{array}{l}\text { Duração: } 6 \text { meses. Descrição: } \\
\text { foram utilizados painéis } \\
\text { coloridos, brincadeiras, } \\
\text { transparências, fantoches, } \\
\text { vídeos e figuras de alimentos } \\
\text { com os escolares }\end{array}$ & $\begin{array}{c}\text { Instrumento: questionário } \\
\text { desenvolvido para o } \\
\text { estudo, IPAQ e avaliação } \\
\text { antropométrica. Variáveis } \\
\text { analisadas: nível de } \\
\text { conhecimento em alimentação } \\
\text { e hábitos alimentares } \\
\text { (subamostra de } 14,9 \% \text { do } \\
\text { total), bem como o NAF } \\
\text { (subamostra de } 12,6 \% \text { do total) }\end{array}$ & $\begin{array}{l}\text { Aumento: pontuação média } \\
\text { para o nível de conhecimento } \\
\text { em alimentação e práticas } \\
\text { alimentares (valores não } \\
\text { apresentados). Média do IMC } \\
\text { após intervenção }\left(0,97 \mathrm{~kg} / \mathrm{m}^{2}\right. \\
\text { nos meninos e } 1,03 \mathrm{~kg} / \mathrm{m}^{2} \text { nas } \\
\text { meninas) }\end{array}$ \\
\hline Gabriel et al. 13 & $\begin{array}{l}\text { Avaliar os resultados de um } \\
\text { programa de intervenção } \\
\text { nutricional em escolares do } \\
\text { Ensino Fundamental }\end{array}$ & $\begin{array}{c}\text { Duração: } 2 \text { meses. Descrição: } \\
\text { palestras e atividades } \\
\text { educativas sobre o Guia da } \\
\text { Pirâmide Alimentar, montagem } \\
\text { de cardápio e reciclagem do } \\
\text { lixo }\end{array}$ & $\begin{array}{l}\text { Instrumento: questionário } \\
\text { desenvolvido para o estudo } \\
\text { e avaliação antropométrica. } \\
\text { Variáveis analisadas: IMC e } \\
\text { hábitos alimentares }\end{array}$ & $\begin{array}{c}\text { Redução: } 12,6 \% \text { na proporção } \\
\text { de meninos que trazem de } \\
\text { casa bolachas recheadas na } \\
\text { escola particular. Aumento: } \\
21,6 \% \text { e 10,5\% na proporção } \\
\text { de meninos e meninas que } \\
\text { preferem consumir frutas; } \\
\text { 15,0\% na proporção de } \\
\text { escolares que consomem } \\
\text { merenda escolar na escola } \\
\text { pública }\end{array}$ \\
\hline
\end{tabular}

(continua) 
Tabela 2 (continuação)

\begin{tabular}{|c|c|c|c|c|}
\hline Referência & Objetivo & Características da intervenção & Variáveis & Principais resultados \\
\hline Sichieri et al. 14 & $\begin{array}{l}\text { Determinar se um programa } \\
\text { educacional focado na redução } \\
\text { do consumo de bebidas } \\
\text { com adição de açúcar pode } \\
\text { prevenir o ganho de peso } \\
\text { excessivo em escolares }\end{array}$ & $\begin{array}{l}\text { Duração: } 7 \text { meses. Descrição: } \\
\text { atividades educacionais, } \\
\text { produção de material } \\
\text { educativo e estímulo ao } \\
\text { consumo de água entre os } \\
\text { escolares }\end{array}$ & $\begin{array}{l}\text { Instrumento: recordatório } \\
\text { alimentar de } 24 \text { h e avaliação } \\
\text { antropométrica. Variáveis } \\
\text { analisadas: IMC e a ingestão } \\
\text { alimentar }\end{array}$ & $\begin{array}{l}\text { Redução: ingestão média de } \\
\text { refrigerantes em cada turma } \\
\text { escolar (-69 ml/dia) após } \\
\text { intervenção; ingestão diária de } \\
\text { bebidas gaseificadas (-63mL/ } \\
\text { dia) após intervenção }\end{array}$ \\
\hline Vargas 18 & $\begin{array}{c}\text { Investigar o efeito de um } \\
\text { programa para prevenção } \\
\text { da obesidade baseado } \\
\text { em educação nutricional e } \\
\text { incentivo à prática de atividade } \\
\text { física }\end{array}$ & $\begin{array}{l}\text { Duração: } 4 \text { meses. Descrição: } \\
\text { educação nutricional (palestras, } \\
\text { vídeos, dinâmicas e oficinas) e } \\
\text { incentivo à prática de atividade } \\
\text { física (cartazes e olimpíadas } \\
\text { escolares) }\end{array}$ & $\begin{array}{l}\text { Instrumentos: questionário } \\
\text { desenvolvido para o estudo } \\
\text { e avaliação antropométrica. } \\
\text { Variáveis analisadas: } \\
\text { maturação, IMC e hábitos } \\
\text { alimentares }\end{array}$ & $\begin{array}{l}83,8 \% \text { dos participantes } \\
\text { relataram uma maior animação } \\
\text { para prática de atividade física } \\
\text { após intervenção. Aumento: } \\
13,9 \% \text { na proporção de } \\
\text { escolares que não compram } \\
\text { lanches vendidos por } \\
\text { ambulantes }\end{array}$ \\
\hline Zancul 22 & $\begin{array}{l}\text { Analisar os reflexos de um } \\
\text { programa de educação } \\
\text { nutricional nas condutas } \\
\text { alimentares de escolares }\end{array}$ & $\begin{array}{l}\text { Duração: } 6 \text { meses. Descrição: } \\
\text { Apresentação de filmes, } \\
\text { elaboração de cartazes, } \\
\text { dramatização, leitura de textos, } \\
\text { aplicação de jogos e dinâmicas }\end{array}$ & $\begin{array}{l}\text { Instrumento: questionário } \\
\text { desenvolvido para o estudo } \\
\text { e avaliação antropométrica. } \\
\text { Variáveis analisadas: IMC, } \\
\text { hábitos alimentares e } \\
\text { conhecimento nutricional }\end{array}$ & $\begin{array}{l}\text { Aumento: } 33,3 \% \text { na proporção } \\
\text { de escolares que referiram } \\
\text { consumir desjejum todos } \\
\text { os dias; } 30,9 \text { e } 21,5 \% \text { na } \\
\text { proporção de adolescentes } \\
\text { que referiram consumir } \\
\text { verduras cruas no almoço e } \\
\text { jantar, respectivamente }\end{array}$ \\
\hline Barros et al. 15 & $\begin{array}{l}\text { Avaliar a eficácia de um } \\
\text { programa de intervenção em } \\
\text { estudantes do Ensino Médio } \\
\text { do Projeto Saúde na Boa }\end{array}$ & $\begin{array}{l}\text { Duração: } 9 \text { meses. Descrição: } \\
\text { divulgação de pôsteres e } \\
\text { cartilhas para discussões em } \\
\text { sala de aula. Oportunidades } \\
\text { ambientais organizadas } \\
\text { (passeios de bicicleta, dia da } \\
\text { fruta etc.), kits (US\$ 500) para } \\
\text { adquirir equipamentos de } \\
\text { educação física para as escolas }\end{array}$ & $\begin{array}{c}\text { Instrumentos: questionário } \\
\text { desenvolvido para o estudo. } \\
\text { Variáveis analisadas: prática de } \\
\text { atividade física }\end{array}$ & $\begin{array}{l}\text { Redução: 3,6\% na prevalência } \\
\text { de inatividade física após } \\
\text { intervenção. Aumento: } \\
\text { número de dias que o Gl } \\
\text { segue a recomendação de } \\
60 \text { minutos/dia de atividade } \\
\text { física moderada a vigorosa, em } \\
\text { comparação com o GC, após } \\
\text { intervenção }\end{array}$ \\
\hline Cavalcanti 19 & $\begin{array}{l}\text { Verificar os efeitos de uma } \\
\text { intervenção para a promoção } \\
\text { de hábitos alimentares } \\
\text { saudáveis entre os escolares }\end{array}$ & $\begin{array}{l}\text { Duração: NI. Descrição: } \\
\text { programa educativo (palestras, } \\
\text { oficinas, jogos, orientação } \\
\text { nutricional aos familiares) } \\
\text { focando atividade física e } \\
\text { hábitos alimentares }\end{array}$ & $\begin{array}{l}\text { Instrumentos: questionário } \\
\text { desenvolvido para o estudo } \\
\text { e avaliação antropométrica. } \\
\text { Variáveis analisadas: IMC, } \\
\text { percentual de gordura corporal } \\
\text { e hábitos alimentares }\end{array}$ & $\begin{array}{l}\text { Aumento: fator de proteção } \\
\text { (razão de chance = 0,47) nas } \\
\text { crianças do Gl para apresentar } \\
\text { hábitos alimentares saudáveis, } \\
\text { em comparação ao GC }\end{array}$ \\
\hline Farias et al. 16 & $\begin{array}{l}\text { Avaliar o efeito da atividade } \\
\text { física programada na } \\
\text { composição corporal dos } \\
\text { escolares }\end{array}$ & $\begin{array}{l}\text { Duração: } 11 \text { meses. Descrição: } \\
\text { duas aulas semanais (60 } \\
\text { minutos) com atividade } \\
\text { física durante as aulas de } \\
\text { educação física escolar, } \\
\text { baseando-se em atividades } \\
\text { aeróbias (caminhadas, corridas } \\
\text { alternadas), jogos esportivos e } \\
\text { alongamento }\end{array}$ & $\begin{array}{c}\text { Instrumentos: avaliação } \\
\text { antropométrica. Variáveis } \\
\text { analisadas: IMC, dobras } \\
\text { cutâneas, circunferências, } \\
\text { percentual de gordura corporal } \\
\text { e massa corporal gorda e } \\
\text { magra }\end{array}$ & $\begin{array}{c}\text { Redução: } 4,3 \% \text { na proporção } \\
\text { de escolares obesos do } \\
\text { Gl; Dobra cutânea tricipital } \\
\text { (-1,6mm e -1,8mm) e } \\
\text { percentual de gordura ( } 1,5 \% \text { e } \\
\text { 1,7\%) nos meninos e meninas } \\
\text { do Gl. Aumento: massa } \\
\text { corporal magra (2,8kg nos } \\
\text { meninos e } 2,4 \text { kg nas meninas) } \\
\text { após a intervenção }\end{array}$ \\
\hline
\end{tabular}

(continua) 
Tabela 2 (continuação)

\begin{tabular}{|c|c|c|c|c|}
\hline Referência & Objetivo & Características da intervenção & Variáveis & Principais resultados \\
\hline Fernandes et al. 17 & $\begin{array}{c}\text { Avaliar o efeito de um } \\
\text { programa de educação } \\
\text { nutricional na prevalência } \\
\text { de sobrepeso/obesidade e } \\
\text { nos hábitos alimentares de } \\
\text { escolares }\end{array}$ & $\begin{array}{l}\text { Duração: } 4 \text { meses. Descrição: } \\
\text { atividades lúdico-educativas, } \\
\text { jogos, teatros de fantoches, } \\
\text { cartazes, brincadeiras, músicas } \\
\text { e histórias infantis }\end{array}$ & $\begin{array}{c}\text { Instrumento: registro } \\
\text { alimentar de três dias e } \\
\text { avaliação antropométrica. } \\
\text { Variáveis analisadas: IMC e } \\
\text { hábitos alimentares durante a } \\
\text { permanência na escola }\end{array}$ & $\begin{array}{l}\text { Redução: } 4,81 \% \text { na proporção } \\
\text { de adolescentes que referiram } \\
\text { consumir suco artificial na } \\
\text { escola em } 2 \text { ou } 3 \text { dias }\end{array}$ \\
\hline Ribeiro 20 & $\begin{array}{l}\text { Verificar o efeito de dois } \\
\text { programas de intervenção no } \\
\text { NAF em adolescentes da rede } \\
\text { pública de ensino }\end{array}$ & $\begin{array}{l}\text { Duração: } 4 \text { meses. Descrição: } \\
\mathrm{GI}_{1} \text { - um encontro semanal } \\
\text { com debates, discussões, } \\
\text { dinâmicas, vivências práticas } \\
\text { para promover atividade } \\
\text { física no cotidiano; } \mathrm{Gl}_{2} \text { - duas } \\
\text { sessões semanais de atividade } \\
\text { física programada compostas } \\
\text { por exercícios aeróbios, de } \\
\text { força e flexibilidade }\end{array}$ & $\begin{array}{l}\text { Instrumentos: questionário } \\
\text { desenvolvido para o estudo } \\
\text { e avaliação antropométrica. } \\
\text { Variáveis analisadas: IMC, } \\
\text { prática de atividade física e } \\
\text { comportamento sedentário }\end{array}$ & $\begin{array}{l}\text { Aumento: tempo de prática } \\
\text { de esportes/exercícios físicos } \\
\text { após a intervenção do } \mathrm{GI}_{1} \\
(359,4 \text { minutos/semana) e do } \\
\mathrm{GI}_{2}(606,4 \text { minutos/semana); } \\
\text { tempo de prática de atividade } \\
\text { física total após a intervenção } \\
\text { do } \mathrm{Gl}_{1}(380,2 \text { minutos/semana) } \\
\text { e do } \mathrm{GI}_{2} \text { (605,8 minutos/ } \\
\text { semana) }\end{array}$ \\
\hline
\end{tabular}

IMC: índice de massa corporal; IPAQ: Questionário Internacional de Atividade Física; GC: grupo controle; Gl: grupo intervenção; Gl ${ }_{1}$ : intervenção sem prática de atividade física; $\mathrm{Gl}_{2}$ : intervenção com atividade física; NAF: nível de atividade física; NI: não indicado.

intervenção. Esses resultados continuaram elevados oito meses após o final do programa. Diante disso, os autores destacam a importância de um trabalho contínuo de educação alimentar e nutricional dentro das escolas, visando à permanência de hábitos alimentares mais saudáveis e à promoção de conhecimento sobre saúde entre os escolares 22 .

Outros programas evidenciaram a importância das ações de intervenção para o desenvolvimento do conhecimento alimentar e de atitudes mais saudáveis entre os escolares, como parte de um processo cognitivo: o aumento significativo ( $p<0,05)$ do nível de conhecimento dos estudantes acerca de hábitos alimentares saudáveis (as pontuações obtidas com o preenchimento dos questionários não foram apresentadas no estudo) 12 e o aumento da porcentagem de escolares que consomem mais frutas no cotidiano após a intervenção (21,6\% e 10,5\% entre meninos e meninas, respectivamente) 13 .

Programas de intervenção focados na prática de atividade física, alimentação saudável e/ou estado nutricional

Os programas de intervenção que focaram a promoção da prática de atividade física, alimentação saudável e/ou estado nutricional utilizaram, de maneira geral, ações de intervenção semelhantes às dos estudos apresentados no tópico anterior; jogos e dinâmicas, apresentação de vídeos e palestras com os escolares, e a distribuição e/ou confecção de material educativo foram as ações desenvolvidas em grande parte dos programas de intervenção 15,18,19,20,21,23. Vale destacar que, em alguns programas, essas ações de intervenção também foram realizadas com professores ou funcionários das escolas 15,21,23.

Embora grande parte dos programas abordados neste tópico 15,18,19,20,21,23 tenha focado suas ações de intervenção tanto na promoção de atividade física (passeios de bicicleta, olimpíadas escolares e jogos esportivos) quanto na alimentação saudável (dinâmicas e jogos educacionais), três estudos apresentaram resultados relacionados a somente um destes comportamentos; dois apresentaram apenas os resultados relacionados à atividade física 15,20, enquanto outro estudo focou seus resultados nos hábitos alimentares e no estado nutricional 19.

Um dos programas de intervenção 15 que analisaram sua efetividade em fatores da atividade física foi realizado em duas cidades brasileiras (Florianópolis e Recife), e teve a participação de 474 escolares (515 no GC), de turmas de ensino médio do período noturno (considerado grupo de risco para hábitos inadequados). Para tanto, o programa teve a duração de nove meses e desenvolveu ações de intervenção como a divulgação de pôsteres temáticos, cartilhas para discussões em sala de aula e oportunidades ambientais organizadas como passeios de bicicleta, dia da fruta e kits de educação física para as escolas (US\$ 500 para cada colégio adquirir materiais e equipamentos selecionados pelos estudantes e o pro- 
fessor de educação física). Após a intervenção, os escolares do GC acumulavam 60 minutos ou mais de atividades físicas moderadas/vigorosas em menos dias da semana, quando comparados aos do GI (2,6 vs. 3,3 dias/semana; $\mathrm{p}<0,01)$. A proporção de estudantes que atingiam a recomendação de atividades físicas ( $\geq 5$ dias por semana com 60 minutos ou mais de atividade física moderada/vigorosa) diminuiu nos dois grupos, no entanto, a intervenção foi efetiva em minimizar estas reduções no GI (GI: 41,1 para 33,1\%; GC: 37,7 para $23,7 \%$; $<<0,01$ ). Contudo, a efetividade dessa intervenção em comportamentos e fatores relacionados à alimentação saudável e ao estado nutricional não foi abordada neste estudo 15 .

Vale destacar o programa de intervenção realizado com 995 escolares (1.146 no GC) da Cidade de Pelotas 23 , que analisou sua efetividade em quatro importantes fatores da saúde: atividade física, alimentação saudável, estado nutricional e consumo de cigarros. A intervenção foi baseada no treinamento dos professores (palestras, discussões e vídeos ilustrativos), com enfoque principalmente no tabagismo, e distribuição de material didático para as escolas do GI a ser utilizado pelos professores nos seis meses seguintes. É relevante o fato de que, mesmo após essa intervenção, não foram observadas melhorias significativas nos conhecimentos e atitudes sobre alimentação, no IMC e no nível de atividade física dos adolescentes, sendo verificados resultados significativos somente com os professores (aumento do conhecimento acerca do tabagismo e redução do fumo e do consumo de gordura). A falta de efetividade dessa intervenção na prática de atividade física e na alimentação dos escolares pode ser parcialmente explicada pela ênfase das ações de intervenção nos comportamentos relacionados ao tabagismo 23.

Em contrapartida, outro programa de intervenção 18 que focou sua efetividade no conhecimento e atitudes relacionadas à atividade física $\mathrm{e}$ alimentação, bem como na melhoria do estado nutricional, verificou que $82,4 \%$ dos escolares relataram maior interesse para a adoção de uma alimentação mais saudável, e 83,8\% disseram estar mais animados para a prática de atividade física, após os quatro meses de intervenção. Também foram identificadas mudanças significativas nos hábitos alimentares dos escolares do GI: aumento da proporção de estudantes que relataram não consumir lanches vendidos por ambulantes $(36,7 \%$ vs. $50,6 \%$; $\mathrm{p}=0,02)$ e que não substituíam o almoço (44,5\% vs. $65,2 \%$; p < 0,01) e o jantar $(38,4 \%$ vs. $54,3 \%$; p < 0,01) por lanches. Contudo, esse programa de intervenção também não foi efetivo para ocasionar mudanças positivas no estado nutricional ou no IMC 18.
Apenas dois programas de intervenção promoveram a prática regular de exercícios físicos como ações de intervenção 16,20. Um desses programas 20 foi realizado em São Paulo e teve o propósito de verificar o efeito de dois tipos de intervenção no nível de atividade física de 69 adolescentes (21 no GC). Aintervenção teve a duração de quatro meses, sendo constituída por dois grupos de intervenção: (1) grupo educação em atividade física e saúde: realizou encontros semanais com duração de 60 minutos cada, para discutir sobre atividades físicas e estilo de vida; (2) grupo exercício físico: participou de duas sessões semanais de exercícios físicos estruturados, com duração de 60 minutos e consistiu de exercícios aeróbios, de força e de flexibilidade. Após a intervenção, houve aumento significativo no tempo semanal despendido em esportes/ exercícios físicos (302,6 para 662,0 minutos/semana; 463,2 para 1.069,6 minutos/semana) e na prática de atividade física total (430,0 para 810,2 minutos/semana; 581,2 para 1.187,0 minutos/semana) entre os escolares do grupo educação em atividade física e saúde, e do grupo exercício físico, respectivamente. Já os escolares do GC mantiveram sua prática habitual de atividade física 20 .

\section{Discussão}

Esta revisão sistemática verificou que existem poucos estudos científicos publicados nos últimos cinco anos sobre programas de intervenção em promoção da saúde relacionados à atividade física e/ou hábitos alimentares em escolas brasileiras. Apesar de países norte-americanos e europeus produzirem diversos estudos 8,24,25,26, principalmente Estados Unidos, Inglaterra e França, revisões sistemáticas recentes 8,27 também ressaltaram a pequena quantidade de trabalhos publicados sobre esse tema, mediante a sua relevância para a saúde pública 4,28 .

Nesta revisão, também se observou que, embora os estudos possuam objetivos similares, ou seja, buscam avaliar o efeito de programas de intervenção sobre aspectos relacionados à saúde de escolares, há grande diversidade acerca da população investigada (localização geográfica, faixa etária, tipo de escola, número amostral), da metodologia de intervenção utilizada e das variáveis analisadas, o que dificulta a comparação dos resultados encontrados e suscitam questões relevantes acerca da promoção de atividade física e alimentação saudável nas escolas brasileiras.

Quanto à localização geográfica, a maioria dos estudos (84,6\%) foi realizada nas regiões Sul e Sudeste do país. Esse fato pode ser reflexo das diferenças no desenvolvimento socioeconômico 
entre as regiões do Brasil. Estudos e intervenções necessitam de investimentos e pesquisadores qualificados para serem executados. A produção de instrumentos de avaliação e de intervenção (cartazes, cartilhas, fôlderes), a aquisição de materiais para mensurações físicas e a capacitação de profissionais para atuação em campo aumentam o custo das intervenções, podendo limitar a duração, o número amostral, o local e a qualidade metodológica da intervenção 29. Alguns trabalhos citam ainda a importância de parcerias entre instituições de pesquisa e apoio de órgãos governamentais para que as intervenções possam abranger um maior número de participantes e estender suas ações aos professores, aos familiares e à comunidade 21,23,30 .

A maior parte das intervenções foi realizada em escolas públicas (76,9\%), possivelmente devido à perspectiva de atenderem à população de baixo nível socioeconômico; a baixa educação e renda familiar têm sido associadas com o desenvolvimento de padrões comportamentais específicos que aumentam os riscos de obesidade e DCNT 31,32,33. Entretanto, apesar do tipo de escola (privada ou pública) poder ser usado como variável de aproximação para o nível socioeconômico, é importante que estudos futuros incluam dados sobre a condição socioeconômica da população estudada. Apenas três trabalhos desta revisão reportaram a condição socioeconômica de seus participantes 14,16,23.

Ao analisar o período de intervenção dos estudos desta revisão, observou-se que $50 \%$ das intervenções tiveram duração inferior a seis meses. Apesar do curto período de intervenção, alguns programas encontraram importantes resultados; como exemplo, Gabriel et al. 13 verificaram na escola privada redução significativa dos porcentuais de bolachas recheadas trazidas de casa pelos meninos e, na escola pública, o aumento significativo do consumo da merenda escolar e da aceitação de frutas, após os dois meses de intervenção. Por outro lado, programas de maior duração (seis meses) 12,22,23 encontraram pouca efetividade das intervenções realizadas na prática de atividade física e na adoção de hábitos alimentares saudáveis. Dessa forma, é complexo afirmar, pelos resultados dos programas aqui apresentados, a influência do período da intervenção sobre os componentes relacionados à saúde dos escolares, visto que a efetividade das intervenções foi independente da duração dos programas. No entanto, a literatura recomenda que programas de promoção com mudanças sustentáveis no estilo de vida de crianças e adolescentes tenham duração prolongada, propiciando aos participantes maiores benefícios com o programa 34,35 .
Quanto ao foco das intervenções, 46,2\% dos estudos intervieram somente em alimentação, $7,7 \%$ apenas em atividade física, e outros $46,2 \%$ intervieram em ambos os comportamentos; estas características dificultaram a análise mais complexa dos resultados destes estudos. Outros fatores que dificultam a comparação entre os estudos e a generalização dos resultados encontrados são: a falta de, ou a descrição insuficiente quanto ao processo de seleção e o número amostral, a distribuição não aleatória entre os GI e GC e, em alguns casos, a própria falta de GC e de pareamento quanto a variáveis intervenientes.

Além disso, outras variáveis relacionadas à prática de atividade física e hábitos alimentares, como comportamento sedentário, condições socioeconômicas e comportamento familiar, foram analisados em poucos estudos ou não foram avaliados. O IMC e o nível de atividade física (NAF) também não foram investigados em todos os estudos, sendo estas duas variáveis de suma importância para análise dos impactos de programas de intervenção 28,36,37 em promoção da saúde. Essas variáveis foram analisadas simultaneamente em apenas três estudos 12,20,23, enquanto a maioria $12,13,14,16,17,18,19,22$ analisou somente o IMC.

O IMC é uma medida de fácil aplicação em estudos populacionais, mundialmente aceito como indicador antropométrico de desnutrição e de sobrepeso/obesidade 38,39,40. Contudo, dos oito trabalhos que utilizaram o IMC como indicador antropométrico, apenas um 16 apresentou redução na proporção de obesidade como um resultado significativo das ações de intervenção.

O NAF é um indicador quantitativo da atividade física habitual e pode ser estimado usando-se diversos métodos (questionários, monitoração da frequência cardíaca, acelerômetros e pedômetros), de acordo com a viabilidade de cada estudo. Iniciativas de promoção da saúde deveriam objetivar o aumento do NAF de maneira sustentável 41. Alguns programas internacionais de intervenção em escolas observaram melhoras significativas no NAF durante as aulas de educação física 42 ou na vida diária 25,27,43, principalmente em subgrupos de maior risco ao sedentarismo ${ }^{44}$.

A mudança nos hábitos alimentares foi um dos resultados mais reportados pelos estudos com foco na alimentação saudável 11,12,13,14,17,22, pela diminuição do consumo de alimentos com alto valor calórico e/ou aumento da ingestão de frutas e verduras. Uma revisão dos programas de intervenção nutricional de diferentes países 45 também relatou importantes mudanças de hábitos alimentares utilizando-se programas de educação nutricional. 
Dos estudos que tiveram atividade física e alimentação como foco da intervenção, um identificou o aumento no tempo semanal de atividade física 20 , enquanto os demais estudos mostraram resultados bastante diferenciados, a saber: redução da falta de vontade e falta de energia para prática de atividade física 18 , redução da inatividade física no grupo intervenção 15 , não substituição do almoço e jantar por lanches 18, e a melhoria dos hábitos e informações sobre alimentação $18,19,21$. Um programa 46 que investigou os efeitos de intervenções combinadas com atividade física e alimentação em 522 escolares chilenos verificou, após cinco meses de intervenção, tendência na diminuição da obesidade entre os préescolares e uma melhora significativa da aptidão física por meio do teste de corrida (6 minutos). Gorely et al. 25 também focaram sua pesquisa na atividade física e na alimentação, e verificaram o aumento do NAF e do número de passos diários após onze meses de intervenção. Mesmo não tendo encontrado alteração no consumo de frutas e vegetais, os autores consideraram benéficos os efeitos de programas que utilizam a atividade física e a alimentação como estratégia para mudança no estilo de vida e prevenção da obesidade em crianças e adolescentes 25 .

A atividade física programada tem sido um dos procedimentos mais empregados para o tratamento da obesidade. Vízcaíno et al. 47 verificaram redução na adiposidade (IMC e dobra cutânea tricipital) de 513 crianças após seis meses em um programa de intervenção em atividade física (três aulas de 90 minutos por semana). Outro estudo 24 também verificou melhora na composição corporal, principalmente entre as crianças que apresentavam obesidade. Não obstante, trabalhos prévios que enfatizam a prática de atividade física como ações de intervenção para crianças e adolescentes obesos, também mostraram melhoria na composição corporal destes indivíduos 47,48,49. Contudo, deve-se salientar que os mecanismos de ação e otimização dos programas de atividade física necessitam de melhores esclarecimentos devido às divergências encontradas (intensidade e duração do exercício, protocolos de avaliação antropométrica e composição corporal) entre os diversos estudos 48. Nesta revisão, os trabalhos que promoveram a prática regular de atividade física como ações de intervenção evidenciaram melhoras principalmente na composição corporal 16 e na prática semanal de atividade física 20 entre os escolares.

Existem fortes evidências mostrando que a educação física escolar pode ser uma estratégia efetiva para aumentar a atividade física entre estudantes 20 , principalmente no Brasil, onde a educação física escolar é obrigatória 50 . Na mesma direção, a alimentação escolar é oferecida gratuitamente em grande parte das escolas públicas do país, o que poderia ser melhor aproveitado para a promoção de hábitos alimentares saudáveis 13,17. Entretanto, algumas barreiras (profissionais desmotivados e/ou despreparados, falta de recursos financeiros, físicos e materiais, e estrutura curricular desatualizada) precisariam ser superadas para que as escolas ofereçam uma educação física e alimentação de qualidade e em sintonia com os objetivos da promoção de saúde 50 . Nesse contexto, a escola pode surgir como um ambiente privilegiado para o desenvolvimento e a aprendizagem de competências em saúde como um conceito estruturador, incluindo também o envolvimento de diversos níveis de gestão, a formação de professores, e adaptações curriculares, ajudando a definir uma política sustentável para a saúde na comunidade escolar.

Por fim, é também fundamental que as intervenções realizadas sejam sistematizadas, acompanhadas e avaliadas, e seus resultados disseminados. Apesar das dificuldades de natureza operacional envolvidas nesses processos (dificuldade para medir comportamentos e fatores da saúde, selecionar uma população-controle semelhante à população-alvo, acompanhar as mudanças graduais e em longo prazo, dentre outros), a superação possibilitará determinar quais as ações de intervenção podem ser mais efetivas no contexto brasileiro.

\section{Conclusão}

As elevadas taxas de obesidade, atividade física insuficiente e má alimentação em crianças e adolescentes brasileiros representam uma oportunidade para a realização de intervenções que buscam a modificação deste quadro e a promoção da saúde. Entretanto, essas ações, mesmo que estejam sendo desenvolvidas, não são devidamente documentadas e avaliadas. Os poucos estudos publicados de intervenção em atividade física e alimentação saudável em escolares, bem como algumas deficiências metodológicas entre os programas de intervenção, dificultaram a avaliação da efetividade destas ações. Entretanto, todos os trabalhos nesta revisão reportaram alguma alteração positiva após as intervenções, mesmo que não estatisticamente significativa, demonstrando a potencialidade destes programas para a promoção de uma vida mais saudável.

Espera-se que os resultados apresentados e as críticas produzidas nesta revisão sirvam para estimular a sistematização e publicação de infor- 
mações e a melhora da qualidade metodológica de pesquisas sobre programas de intervenção em saúde nas escolas, produzindo eventualmente mais evidências científicas que auxiliem a obtenção de maior qualidade de vida e saúde na população brasileira.

\section{Resumo}

Este artigo é uma revisão sistemática da literatura sobre intervenções em atividade física elou alimentação saudável em escolares brasileiros. Estudos completos publicados entre 2004 e 2009 foram buscados nas bases eletrônicas SciELO, MEDLINE e no portal da CAPES, nas referências dos artigos encontrados e por contato com autores. Seis estudos focaram a intervenção nos componentes alimentares, outros seis trataram tanto da alimentação quanto da prática de atividade física, e um estudo focou modificações na composição corporal. As intervenções tiveram resultados diversos de acordo com seus objetivos: aumento da quantidade semanal de atividade física; melhoria dos hábitos $e$ conhecimentos sobre alimentação; e redução da prevalência de sobrepeso e obesidade. Programas de promoção da saúde nas escolas são fundamentais para aumentar a conscientização sobre a importância da promoção da saúde e para a adoção de hábitos saudáveis. Entretanto, há a necessidade de mais estudos longitudinais que gerem evidências sobre a sustentabilidade dos programas e dos hábitos saudáveis desenvolvidos.

Atividade Física; Nutrição em Saúde Pública; Estudantes

\section{Colaboradores}

E. A. Souza elaborou a proposta do estudo, participou de todas as etapas das análises de dados, interpretação e discussão dos resultados, foi responsável por toda a revisão bibliográfica e pela elaboração de todas as versões do manuscrito. V. C. Barbosa Filho participou da elaboração da proposta do estudo, colaborou no gerenciamento dos dados e em todas as etapas da análise dos dados, na discussão dos resultados e na revisão de todas as versões do manuscrito. J. A. D. Nogueira revisou o trabalho desde a sua concepção até as fases de análise e redação. M. R. Azevedo Júnior colaborou na elaboração na revisão crítica do conteúdo e na aprovação da versão final do artigo. 


\section{Referências}

1. Langness A, Richter M, Hurrelmann K. Subjektives Wohlbefinden im internationalen Vergleich. Ergebnisse der "Health Behaviour in School-aged Children”-Studie. Psychomed 2005; 4:196-204.

2. Dowdell EB, Santucci ME. Health risk behavior assessment: nutrition, weight, and tobacco use in one urban seventh-grade class. Public Health Nurs 2004; 21:128-36.

3. Instituto Brasileiro de Geografia e Estatística. Pesquisa Nacional de Saúde do Escolar, 2009. Rio de Janeiro: Instituto Brasileiro de Geografia e Estatística; 2009.

4. Ministério da Saúde. Escolas promotoras de saúde: experiências do Brasil. Brasília: Ministério da Saúde; 2006. (Série Promoção da Saúde, 6).

5. Hallal PC, Bertoldi AD, Gonçalves H, Victora CG. Prevalência de sedentarismo e fatores associados em adolescentes de 10-12 anos de idade. Cad Saúde Pública 2006; 22:1277-87.

6. Verstraete S, Cardon G, De Clercq DLR, De Bourdeaudhuij IMM. A comprehensive physical activity promotion programme at elementary school: the effects on physical activity, physical fitness and psychosocial correlates of physical activity. Public Health Nutr 2007; 10:477-84.

7. Peterson KE, Fox MK. Addressing the epidemic of childhood obesity through school-based interventions: what has been done and where do we go from here? J Law Med Ethics 2007; 35:113-30.

8. Sharma M. International school-based interventions for preventing obesity in children. Obes Rev 2006; 8:155-67.

9. Sharma M. School-based interventions for childhood and adolescent obesity. Obes Rev 2006; 7:261-9.

10. Nahas MV, Barros MVG, Assis MAA, Hallal PC, Florindo AA, Konrad L. Methods and participant characteristics of a randomized intervention to promote physical activity and healthy eating among Brazilian high school students: The Saúde na Boa Project. J Phys Act Health 2009; 6:153-62.

11. Gaglianone CP, Taddei JAAC, Colugnati FAB, Magalhães CG, Davanço GM, Macedo L, et al. Nutrition education in public elementary schools of São Paulo, Brazil: the reducing risks of illness and death in adulthood project. Rev Nutr 2006; 19:309-20.

12. Deminice R, Laus MF, Marins TM, Silveira SDO, Dutra de Oliveira JE. Impacto de um programa de educação alimentar sobre conhecimentos, práticas alimentares e estado nutricional de escolares. Alimentos e Nutrição 2007; 18:35-40.

13. Gabriel CG, Santos MV, Vasconcelos FAG. Avaliação de um programa para promoção de hábitos alimentares saudáveis em escolares de Florianópolis, Santa Catarina, Brasil. Rev Bras Saúde Matern Infant 2008; 8:299-308.

14. Sichieri R, Trotte AP, Souza RA, Veiga GV. School randomized trial on prevention of excessive weight gain by discouraging students from drinking sodas. Public Health Nutr 2008; 12:197-202.
15. Barros MVG, Nahas MV, Hallal PC, Farias Júnior JC, Florindo AA, Barros SSH. Effectiveness of a school-based intervention on physical activity for high school students in Brazil: The Saúde na Boa Project. J Phys Act Health 2009; 6:163-9.

16. Farias ES, Paula F, Carvalho WRG, Gonçalves EM, Baldin AD, Guerra Júnior G. Efeito da atividade física programada sobre a composição corporal em escolares adolescentes. J Pediatr (Rio J) 2009; 85:28-34.

17. Fernandes PS, Bernardo CO, Campos RMMB, Vasconcelos FAG. Evaluating the effect of nutritional education on the prevalence of overweight/ obesity and on foods eaten at primary schools. J Pediatr (Rio J) 2009; 85:315-21.

18. Vargas ICS. Implementação de um programa de prevenção de obesidade para adolescentes em ambiente escolar - efeitos sobre medidas antropométricas e práticas alimentares [Dissertação de Mestrado]. Rio de Janeiro: Centro de Ciências da Saúde, Universidade Federal do Rio de Janeiro; 2008.

19. Cavalcanti LA. Efeitos de uma intervenção em escolares do ensino fundamental I, para a promoção de hábitos alimentares saudáveis [Dissertação de Mestrado]. Taguatinga: Faculdade de Educação Física, Universidade Católica de Brasília; 2009.

20. Ribeiro EHC. Efeito de dois programas de intervenção no nível de atividade física de adolescentes matriculados em escolas da rede pública de ensino da Zona Leste da cidade de São Paulo, SP [Dissertação de Mestrado]. São Paulo: Faculdade de Saúde Pública, Universidade de São Paulo; 2009.

21. Barros MVG. Atividades físicas e padrão de consumo alimentar em estudantes do ensino médio em Santa Catarina [Tese de Doutorado]. Porto Alegre: Escola Superior de Educação Física, Universidade Federal do Rio Grande do Sul; 2004.

22. Zancul MS. Orientação nutricional e alimentar dentro da escola: Formação de conceitos e mudanças de comportamento [Tese de Doutorado]. Araraquara: Faculdade de Ciências Farmacêuticas, Universidade Estadual Paulista; 2008.

23. Menezes A, Assunção MC, Neutzling M, Malcon M, Hallal PC, Marques A, et al. Effectiveness of an educational intervention on smoking, diet and physical activity among adolescents. Pelotas: Universidade Federal de Pelotas; 2006. (Final report).

24. Lazaar N, Aucouturier J, Ratel S, Rance M, Meyer $\mathrm{M}$, Duché P. Effect of physical activity intervention on body composition in young children: influence of body mass index status and gender. Acta Paediatr 2007; 96:1315-20.

25. Gorely T, Nevill ME, Morris JG, Stensel DJ, Nevill A. Effect of a school-based intervention to promote healthy lifestyles in 7-11 year old children. Int J Behav Nutr Phys Act 2009; 6:5-17.

26. World Health Organization. The challenge of obesity in the WHO European Region and the strategies for response. Geneva: World Health Organization; 2007. 
27. Doak C, Visscher T, Renders C, Seidell J. The prevention of overweight and obesity in children and adolescents: a review of interventions and programmes. Obes Rev 2006; 7:111-36.

28. World Health Organization. Global strategy on diet, physical activity and health. Fifty-seventh World Health Assembly. Provisional agenda item 12.6 (A57/9), 2004. http://apps.who.int/gb/ebwha/pdf_ files/WHA57/A57_9-en.pdf (acessado em 20/Dez/ 2009).

29. Jones SE, Brener ND, McManus T. Prevalence of school policies, programs, and facilities that promote a healthy physical school environment. Am J Public Health 2003; 93:1570-5.

30. Bauman A, Craig CL. The place of physical activity in the WHO Global Strategy on diet and physical activity. Int J Behav Nutr Phys Act 2005; 2:1-6.

31. Richter M, Erhart M, Vereecken CA, Zambon A, Boyce W, Gabhainn SN. The role of behavioral factors in explaining socio-economic differences in adolescent health: a multilevel study in 33 countries. Soc Sci Med 2009; 69:396-403.

32. Gomes FS, Anjos LA, Vasconcelos MTL. Associação entre o estado nutricional e a situação socioeconômica de adolescentes em Niterói, Rio de Janeiro, Brasil. Cad Saúde Pública 2009; 25:2446-54.

33. Silva GAP, Balaban G, Motta MEFA. Prevalência de sobrepeso e obesidade em crianças e adolescentes de diferentes condições socioeconômicas. Rev Bras Saúde Matern Infant 2005; 5:53-9.

34. Salmon J, Booth M, Phongsavan P, Murphy N, Timperio A. Promoting physical activity participation among children and adolescents. Epidem Rev 2007; 29:144-59.

35. Kamath CC, Vickers KS, Ehrlich A, McGovern L, Johnson, J, Singhal V, et al. Behavioral interventions to prevent childhood obesity: a systematic review and metaanalyses of randomized trials. J Clin Endocrinol Metab 2008; 12:4606-15.

36. Miles L. Physical activity and health. Nutr Bull 2007; 32:314-63.

37. Mastroeni SSBS. Importância da atividade física sobre fatores de risco para o desenvolvimento de doenças cardiovasculares em adolescentes [Tese de Doutorado]. São Paulo: Faculdade de Saúde Pública, Universidade de São Paulo; 2009.

38. Januário RSB, Nascimento MA, Barazetti LK, Reichert FF, Mantoan JPB, Oliveira AR. Índice de massa corporal e dobras cutâneas como indicadores de obesidade em escolares de 8 a 10 anos. Rev Bras Ciênc Mov 2008; 10:266-70.

39. Fernandes RA, Oliveira AR, Freitas Júnior IF. Correlação entre diferentes indicadores de adiposidade corporal e atividade física habitual em jovens do sexo masculino. Rev Bras Cineantropom Desempenho Hum 2006; 8:32-8.
40. Eisenmann JC, Heelan KA, Welk GJ. Assessing body composition among 3- to 8-year-old children: anthropometry, BIA, and DXA. Obes Rev 2004; 12:1633-40.

41. Simon C, Wagner A, DiVita C, Rauscher E, KleinPlatat C, Arveiler D, et al. Intervention centered on adolescents' physical activity and sedentary behaviour (ICAPS): concepts and 6-month results. Int J Obes Relat Metab Disord 2004; 28 Suppl 3:S96-103.

42. Sallis JF, McKenzie TL, Alcaraz JE, Kolody B, Faucette N, Hovell MF. The effects of a 2-year Physical Education Program (SPARK) on physical activity and fitness in elementary school students. Am J Public Health 1997; 87:1328-34.

43. Sallis JF, McKenzie TL, Conway TL, Elder JP, Prochaska JJ, Brown M, et al. Environmental interventions for eating and physical activity a randomized controlled trial in middle schools. Am J Prev Med 2003; 24:209-17.

44. Webber LS, Catellier DJ, Lytle LA, Murray DM, Pratt CA, Young DR, et al. Promoting physical activity in middle school girls trial of activity for adolescent girls. Am J Prev Med 2008; 34:173-84.

45. Jaime PC, Lock K. Do school based food and nutrition policies improve diet and reduce obesity? Prev Med 2009; 48:45-53.

46. Kain J, Concha F, Salazar G, Leyton B, Rodriguez MP, Ceballos X, et al. Prevención de obesidad en preescolares y escolares de escuelas Municipales de una Comuna de Santiago de Chile: proyecto piloto 2006. Arch Lationoam Nutr 2009; 59:139-46.

47. Vízcaino VM, Aguilar FS, Gutiérrez RF, Martínez MS, López MS, Martínez SS, et al. Assessment of an after-school physical activity program to prevent obesity among 9- to 10-year-old children: a cluster randomized trial. Int J Obes 2008; 32:12-22.

48. Sabia RV, Santos JE, Ribeiro RPP. Efeitos da atividade física associada à orientação alimentar em adolescentes obesos: comparação entre o exercício aeróbio e anaeróbio. Rev Bras Med Esporte 2004; 10:349-56.

49. Barbosa Filho VC, Souza EA, Albuquerque AB, Ribeiro EAG. Atividade física na infância: um início para vida saudável. Rev Educ Fís 2007; 18 Suppl 1:263-6.

50. Hoehner CM, Soares J, Perez DP, Ribeiro IC, Joshu CE, Pratt M, et al. Physical activity interventions in Latin America: a systematic review. Am J Prev Med 2008 ; 34:224-33.

Recebido em 17/Jul/2010

Versão final reapresentada em 27/Abr/2011

Aprovado em 18/Mai/2011 